\title{
Prospective randomized comparison of endoscopic submucosal tunnel dissection and conventional submucosal dissection in the resection of superficial esophageal/gastric lesions in a living porcine model
}

Authors

Institutions
Cécile Gomercic $^{1,2}$, Geoffroy Vanbiervliet ${ }^{1,2}$, Jean-Michel Gonzalez ${ }^{2,3}$, Marie-Christine Saint-Paul ${ }^{4}$, Rodrigo Garcès-Duran ${ }^{2,3}$, Emmanuelle Garnier ${ }^{2}$, Xavier Hébuterne ${ }^{1}$, Stéphane Berdah²,5, Marc Barthet ${ }^{2,3}$

Institutions are listed at the end of article.
Bibliography

DOI http://dx.doi.org/

$10.1055 / \mathrm{s}-0034-1393084$

Published online: 8.10.2015

Endoscopy International Open

2015; 03: E577-E583

(c) Georg Thieme Verlag KG

Stuttgart · New York

E-ISSN 2196-9736

\section{Corresponding author}

\section{Geoffroy Vanbiervliet, MD,}

\section{MSc}

Endoscopy unit,

Gastroenterology

L'Archet 2 Hospital

151 Route de Saint Antoine de

Ginestière

CS 23079

06202 Nice cedex 3

France

Phone: 0033 (0)4 92036385

Fax: 0033 (0)4 92035928 vanbiervliet.g@chu-nice.fr
License terms

$(\circledast) \Theta \circledast$
Background and study aims: To assess experimentally endoscopic submucosal tunnel dissection (ESTD) as an alternative technique of endoscopic submucosal resection.

Patients and methods: This was a prospective, randomized, comparative experimental animal study carried out over a period of 9 months at the surgical research and teaching center of AixMarseille University, France. Virtual esophageal and gastric lesions measuring $3 \mathrm{~cm}$ in diameter were resected in pigs weighing 25 to $30 \mathrm{~kg}$. The primary aim was to evaluate ESTD's efficacy compared with endoscopic submucosal dissection (ESD). The secondary aims were to determine complication rates as well as to assess procedure time and procedure speed, histologic quality of the resected specimen, and procedure cost.

Results: Eighteen procedures (9 ESD and 9 ESTD) were performed in nine pigs. The technical suc-

\section{Introduction}

$\nabla$

Technologic advances over recent years in interventional endoscopy have made curative resection of superficial tumors of the digestive tract possible, thus sparing patients from potentially major ablative surgery. Endoscopic submucosal dissection (ESD), which was described at the beginning of the $2000 \mathrm{~s}$, permits $\mathrm{R} 0$ resection of lesions larger than $2 \mathrm{~cm}$ in one single piece. The goal is to obtain healthy lateral and deep resection margins and, so, a lower local recurrence rate [1]. However, ESD presents several disadvantages: The procedure is long, expensive, and technically challenging. At the beginning of the learning process, the adverse events rate is high (it can exceed $50 \%$ ), and execution is poor in terms of en bloc and $\mathrm{R} 0$ resection, restricting its use to centers of expertise [2-5].

These difficulties have led to the development of new techniques like endoscopic submucosal tunnel dissection (ESTD), which was described for cess rate was $88.9 \%$ for both techniques, with one single failure in each. The en bloc resection rate was $100 \%$ for ESTD and $88.9 \%$ for ESD (one failure). The complication rate $(22 \%)$ and median procedure time were similar but dissection speed was quicker with ESTD in the esophagus ( $P=$ 0.03). Median procedure cost (728 Euros for ESD and ESTD) did not differ. On histologic examination, the lateral margins were healthy in $100 \%$ of ESTD and in $88.9 \%$ of ESD $(P=0.49)$. Deep resection margins were of better quality in ESTD (median submucosal thickness: $1307.1 \mu \mathrm{m}$ vs. $884.7 \mu \mathrm{m} ; P=0.039)$.

Conclusions: ESTD is feasible and safe but not superior in the treatment of superficial esophageal/ gastric lesions in porcine models compared with ESD. Nevertheless it provides a better quality histologic specimen.

the first time in humans in 2010 by Inoue et al. for conducting peroral endoscopic myotomy [6]. Then the technique was used for resection of upper gastrointestinal submucosal tumor [7-9]. Recently, it has been shown that ESTD was quick and effective in the resection of large superficial esophageal tumors but also in rescue for ulcerative early gastric cancer resection [10-12]. This method may, therefore, diminish the risk of perforation and hemorrhage through improved visual control during resection and reduce procedure duration. But there are as yet no randomized comparative experimental studies - and, more importantly, there are none in humans - confirming the potential advantages of ESTD compared with conventional ESD.

This study's goal was to experimentally evaluate, in a comparative and randomized fashion, the technical feasibility, efficacy, and safety of ESTD compared with the conventional ESD technique for treating superficial esophageal and gastric tumors in vivo in a porcine animal model. 


\section{Patients and methods}

$\nabla$

\section{Study design}

This was a prospective, comparative, experimental animal study carried out in the surgical research and teaching center of the North campus of the Faculty of Medicine of Aix-Marseille University (Centre d'Enseignement et de Recherche Chirurgicale), France, from November 2012 to July 2013.

The animals used for this study were healthy pigs aged 3 to 4 months weighing 25 to $30 \mathrm{~kg}$. The procedures were conducted under general anesthesia. Once the procedure had been completed, the pigs were euthanized under general anesthesia. The lesions were virtual and corresponded to $3-\mathrm{cm}$ patches of mucosa that were created by marking the border of the lesions electrosurgically using a DualKnife ${ }^{\mathrm{TM}}$. The size of the lesion was determined with the opening of a biopsy forceps. Each pig underwent both submucosal dissection techniques in the antral, fundic, or esophageal areas. The order and site of the procedures were determined by randomization (sealing envelopes drawn at random). This animal experimentation protocol was submitted in regulatory fashion to the local and regional veterinary and sanitary authorities as well as to the animal ethics committee of the surgical research and teaching center of Aix-Marseille University, France, and validated.

The primary goal of this study was to evaluate the efficacy and superiority of ESTD compared with conventional ESD. The primary endpoint was en bloc resection of the demarcated virtual lesion (excision of the virtual lesion in a single specimen, with visualization of all demarcation points on the fragment). Technical success was defined by a resection performed entirely by the dissection technique selected, that either the conventional or tunnel, as described below. Therefore, failure was defined either by the use of an alternative resection method during the procedure (snare or other: technical failure) or by a resection into several fragments (en bloc resection failure). Conversely, the procedure was considered feasible if the resection was complete, in a single piece, and made from beginning to end using the same dissection technique.

The secondary objectives were to determine the safety, length, and cost of the procedures as well as their histopathologic efficacy. The endpoints for these were, respectively, adverse event rates (rates of immediate perforation and hemorrhage); procedure time (timed from the beginning of demarcation to complete fragment dissection) and speed of dissection (lesion surface area divided by procedure time); histologic quality of the resected specimen (macro- and microscopic analysis of lateral and deep resection margins); and the procedure cost (Euros) arising from the use of minor instruments (knives, needle). The size of the lesion for statistical analysis and comparison was evaluated and determined by the pathologist.

\section{Endoscopic equipment}

The endoscope used was a gastroscope (GIF-2TH180, Olympus, Japan) equipped with a transparent cap at its tip (D-201-12704, Olympus, Japan). The submucosal injection was performed using a 23-G needle (NM-4L-1, Olympus, Japan). Incision and dissection were performed using DualKnife ${ }^{\mathrm{TM}}$ (KD-650 L, Olympus, Japan) or ITknife2 ${ }^{\text {TM }}$ (KD-611 L, Olympus, Japan) electrocoagulation scalpels (mode "pulse cut fast", $120 \mathrm{~W}$ ) connected to a generator (ESG-100, Olympus Europe GmbH, Hamburg, Germany). The same instruments were used to coagulate vessels during and at the end of the procedure in soft coagulation mode (power 30 to
$40 \mathrm{~W})$. In the event of hemorrhage, the DualKnife ${ }^{\mathrm{TM}}$ was employed in coagulation mode $(60 \mathrm{~W}$ power - soft coagulation setting). The procedures were performed with room air insufflation.

\section{Dissection technique}

Operators and procedures

ESDs were performed by two interventional endoscopy experts who had conducted more than 50 ESD procedures in humans using the conventional ESD technique. All procedures were filmed and recorded. Before the study began, several preliminary test procedures were performed to practice the ESTD technique. ESTD. First, the lesion's circumference was marked using the DualKnife $^{\mathrm{TM}}$ (power 50W) $\bullet$ Fig. 1. A submucosal injection of a hydroxyethyl-starch solution (Voluven ${ }^{\circledR}$, Fresenius Kabi, Sèvres, France) stained with $0.25 \%$ indigo-carmine solution was then made to lift the lesion for a longer period.

Once the mucosa was lifted, a horizontal incision was made at the distal limit of the lesion and beyond the markings to provide an exit point from the submucosal endoscopic space and so mark the end of the tunnel. Next, a centimeter-sized vertical proximal incision was made approximately $1 \mathrm{~cm}$ above the lesion and exactly in line with it to provide an opening into the submucosal space and so create the tunnel's entry point. The endoscope equipped with a transparent cap was inserted into the submucosa through the proximal opening to create the tunnel. Progression and resection were conducted by means of a combination of two methods: 1) cautiously pushing the endoscope with insufflation, which tears open a space; and 2) dissecting, little by little, the submucosa with the knife until the distal end was reached. The tunneled submucosal space was then enlarged laterally by dissection, and, finally, an incision of the lateral edges of the tumor was made from the distal opening of the tunnel to its proximal end, thus completing resection.

Conventional ESD. After marking and lifting the lesion, a circular mucosal incision was made outside the marker points. Dissection of the submucosal layer was then begun, little by little, starting from the circular incision on the edge and working in toward the center until complete lesion resection was achieved based on a previously described and widely used method [1,13].

\section{Histologic analysis}

At the end of each procedure, the resected fragment was retrieved, stretched, and attached to a slide and then immersed in formaldehyde at $4 \%$ for pathologic analysis. Macroscopic examination consisted of measuring the resection specimens and assessing the lateral resection borders by checking for marker points. The microscopic examination procedure involved paraffin embedding, slices every 3 microns, and hematein-eosin-safran staining. The examination was performed using a Nikon Olympus BX $41^{\mathrm{TM}}$ microscope. Using a Motic ${ }^{\mathrm{TM}}$ camera, submucosal thickness was measured from the muscularis mucosae in the stomach, and from the basement membrane in the esophagus (owing to the thinness of the muscularis mucosae at this level), to the deep resection margin. Two measurements were performed at two different points and then the mean of these two measurements was calculated. Analyses were blindly performed by an experienced pathologist in digestive pathology (Dr. MC Saint-Paul) at the Nice university hospital, France.

\section{Statistical analysis}

The sample size of 18 procedures ( 9 in each group) was calculated using Nquery Advisor ${ }^{\circledR} 7.0$ software sample size calculator, in 

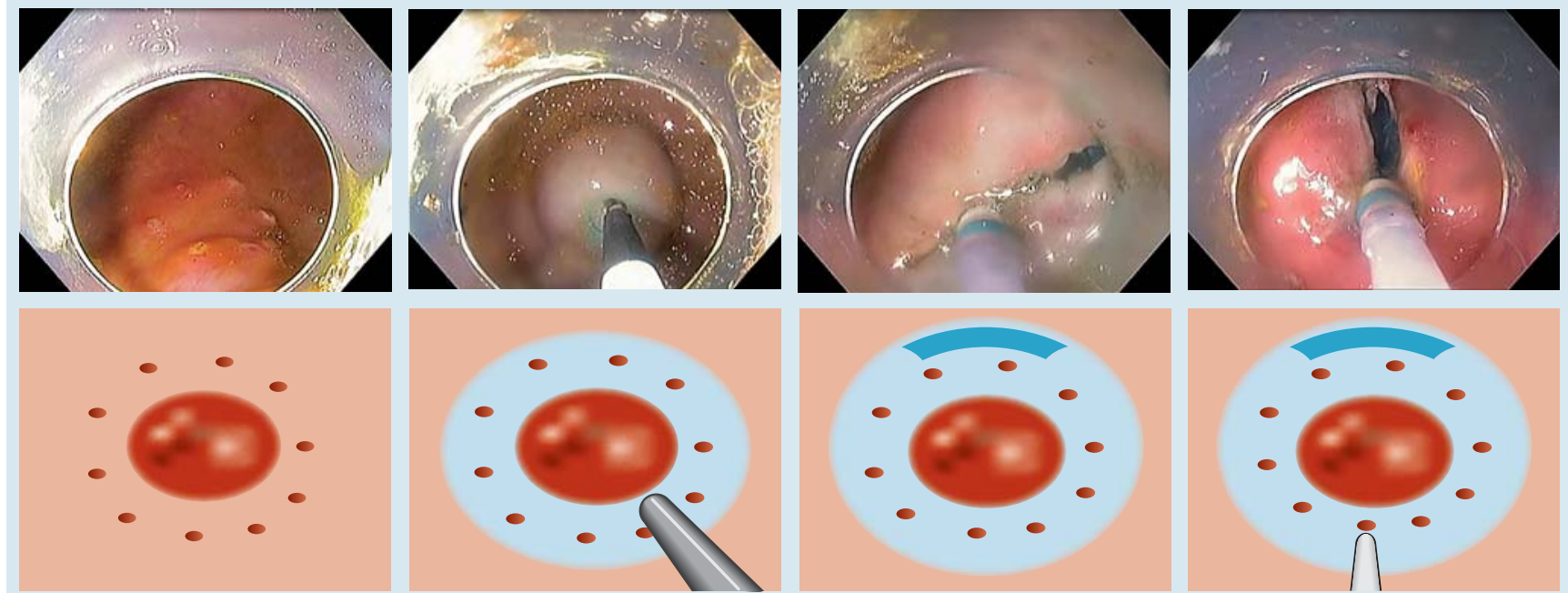

a

b

C
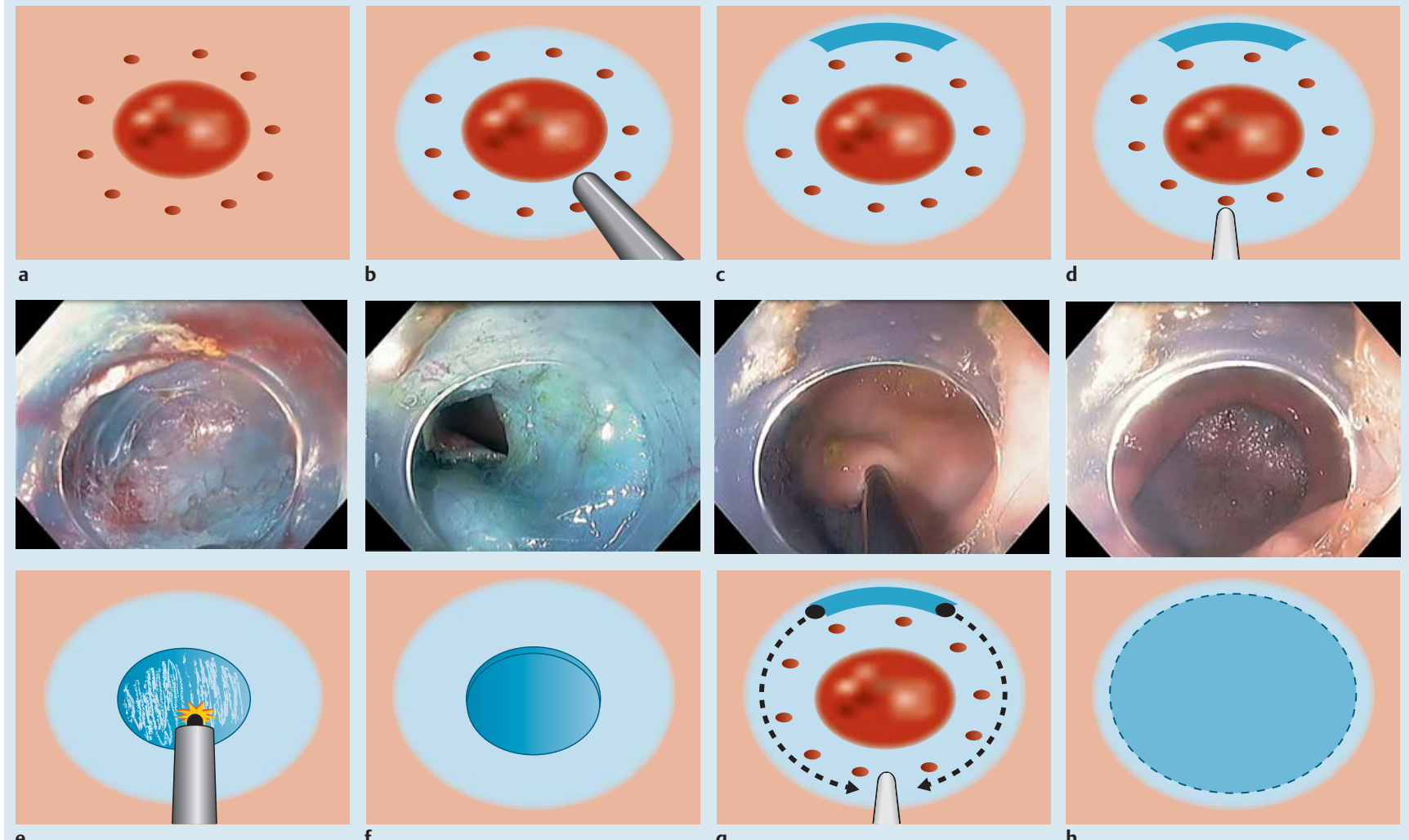

Fig. 1 Different steps in the endoscopic submucosal tunnel dissection method (combined schematic and pictoral views). a Landmarks of the lesion. $\mathbf{b}$ Sub-

g

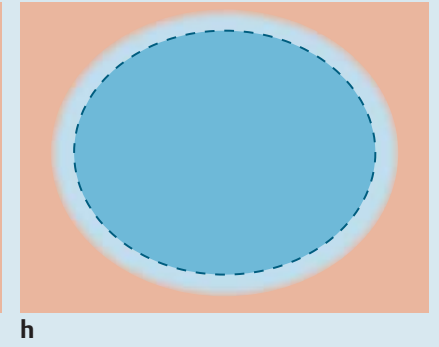
mucosal lifting. c Horizontal distal incision. $\mathbf{d}$ Vertical proximal incision. e Create of the tunnel by the vertical proximal incision. $\mathbf{f}$ Tunneling dissection and reach the horizontal distal incision. $\mathbf{g}$ Incision of the lateral edges. h Final site of resection.

order to detect a $30 \%$ difference in the en bloc resection rate with a Chi2 test and a .05 two-sided significance level associated with an $80 \%$ power. The en bloc resection rate of $70 \%$ for ESD was based on the experience of the operators at the start of the study and we hypothesized a maximal efficacy for ESTD of $100 \%$. The descriptive statistical analysis of quantitative variables was expressed in terms of median, with their standard deviation and range. Qualitative variables were expressed as percentages. Between-variable comparisons were performed by means of the Mann-Whitney test for quantitative variables and by means of Fisher's exact test for qualitative variables. A P value below 0.05 was considered statistically significant. Statistical analyses were performed using the R 2.14.0 software ( $\mathrm{R}$ Development Core Team 2010). The data were compiled in a Microsoft Office Excel 2010 database.

\section{Results}

From November 2012 to July 2013, nine pigs were used as part of this study, giving a total of nine conventional ESDs and nine
ESTDs. The site of resection was antral in five cases (three ESDs and two ESTDs), fundic in five cases (two ESDs and three ESTDs), and esophageal in eight cases (four ESDs and four ESTDs) $\bullet$ Fig. 2. For gastric ESTD and ESD, all procedures were realized with the scope in an antegrade fashion.

The characteristics of the various procedures are summarized in - Table1. The surface area of the lesions treated was comparable in both procedure groups $(P=.18)$. Esophageal resections were statistically smaller than those performed in the stomach $(P<.001)$.

\section{Primary endpoint}

Every procedure was carried out to completion $\bullet$ Table 2. Of the 18 resections performed, 17 (94.4\%) were en bloc resections. The en bloc resection rate was $100 \%$ (9/9) for ESTD and $88.9 \%(8 / 9)$ for $\operatorname{ESD}(P=1)$. The technical success rate was $88.9 \%$ for ESTD and $100 \%$ for $\operatorname{ESD}(P=1)$. In total, feasibility of the two procedures was equivalent ( $88.9 \%$ for ESD and $88.9 \%$ for ESTD) $(P=1)$. 


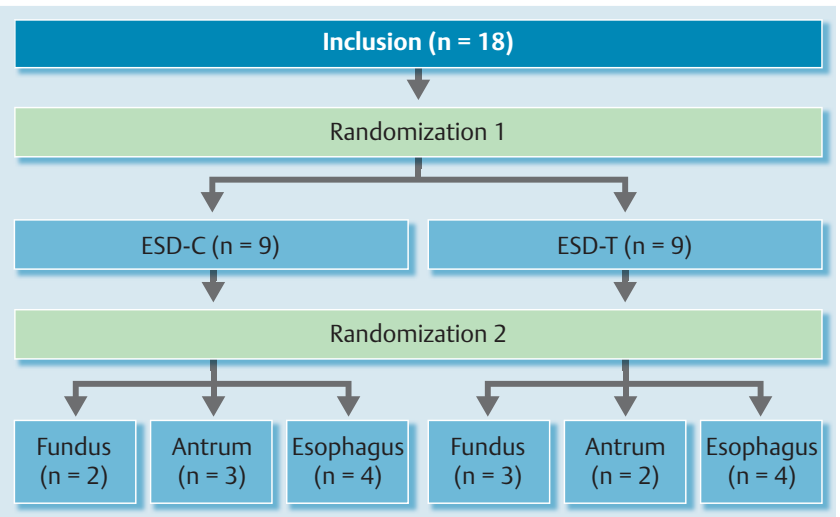

Fig.2 Flow diagram of the randomized procedures.

Table 1 Procedure characteristics: conventional endoscopic submucosal dissection and endoscopic submucosal tunnel dissection.

\begin{tabular}{|llll|} 
& $\begin{array}{l}\text { ESD } \\
\mathbf{n = 9}\end{array}$ & $\begin{array}{l}\text { ESTD } \\
\mathbf{n = 9}\end{array}$ & $\boldsymbol{P}$ \\
\hline $\begin{array}{l}\text { Site, } \mathbf{n} \text { (\%) } \\
\text { Esophagus }\end{array}$ & & \\
Fundus & $4(44.4)$ & $4(44.4)$ & 1 \\
Antrum & $2(22.2)$ & $3(33.3)$ & \\
\hline $\begin{array}{l}\text { Surface area, median } \pm \text { SD } \\
\text { [range], } \mathbf{c m}^{2}\end{array}$ & $3(33.3)$ & $2(22.2)$ & \\
\hline
\end{tabular}

ESD, conventional endoscopic submucosal dissection; ESTD, endoscopic submucosal tunnel dissection

\section{ESTD}

All the ESTD procedures yielded en bloc lesion resection. Submucosal tunneling as well as the distal and proximal incisions was successfully performed in all nine pigs. Only one procedure failed (in antral site), requiring final resection with a snare because of difficulties in cutting one of the edges. The lesion was, however, resected en bloc.

\section{Conventional ESD}

All nine procedures were complete and led to lesion resection, but only eight surgical specimens had macroscopically healthy resection margins (88.9\%). The failed en bloc resection involved a fundic lesion for which all the marker points could not be found on the resection specimen at pathology. This lesion was resected in two pieces.

\section{Secondary endpoints}

Procedure time and speed

Median ESTD time was 30 minutes as against 33 minutes for ESD $(P=.75) \bigcirc$ Table 3. Median dissection speed was $10.7 \mathrm{~mm}^{2} / \mathrm{min}$ for ESD and $10.9 \mathrm{~mm}^{2} / \mathrm{min}$ for ESTD $(P=.34)$. In the esophagus, ESTD was statistically quicker than $\operatorname{ESD}(P=.03)$.

\section{Adverse events}

Perforation rates (22.2\%) were equal for the two techniques Table 2. Two perforations occurred in one esophageal ESD procedure while the submucosal space was being dissected; one occurred with the DualKnife ${ }^{\mathrm{TM}}$ and the other with the ITknife ${ }^{\mathrm{TM}}$.

Table 2 Technical results and costs of procedures.

\begin{tabular}{|c|c|c|c|}
\hline & $\begin{array}{l}\text { ESD } \\
n=9\end{array}$ & $\begin{array}{l}\text { ESTD } \\
n=9\end{array}$ & $P$ \\
\hline Failure, $\mathbf{n}(\%)$ & & & 1 \\
\hline Technical failure & 0 & $1(11.1)$ & \\
\hline En bloc resection failure & $1(11.1)$ & 0 & \\
\hline \multicolumn{4}{|l|}{ Procedure duration, median $\pm S D[$ range], min } \\
\hline Total & $33 \pm 8.8[16-40]$ & $30 \pm 8.9[25-48]$ & .75 \\
\hline Esophagus & $20.5 \pm 8.8[16-36]$ & $25.5 \pm 9.8[23-44]$ & .2 \\
\hline Stomach & $35 \pm 7.3[23-40]$ & $33 \pm 8.8[25-48]$ & 0.6 \\
\hline \multicolumn{4}{|l|}{ Dissection speed, median $\pm \mathrm{SD}$ [range], $\mathrm{mm}^{2} / \mathrm{min}$} \\
\hline Total & $10.7 \pm 8.1[2.2-26]$ & $10.9 \pm 9.1[6.6-26.5]$ & .34 \\
\hline Esophagus & $4.6 \pm 1.6[2.2-6.1]$ & $6.8 \pm 0.12[6.6-6.9]$ & .03 \\
\hline Stomach & $18.2 \pm 6[10.7-26]$ & $24.0 \pm 6.2[10.9-26.5]$ & .1 \\
\hline Perforation, $\mathrm{n}(\%)$ & $2(22.2)$ & $2(22.2)$ & 1 \\
\hline Number of instrument change per procedure, median \pm SD [range], $n$ & $2 \pm 1.5[0-4]$ & $1 \pm 1[0-3]$ & 1 \\
\hline \multicolumn{4}{|l|}{ Number of knifes, $\mathbf{n}(\%)$} \\
\hline 1 & $3(33.3)$ & $2(22.2)$ & .57 \\
\hline 2 & $6(66.6)$ & $7(77.8)$ & \\
\hline Procedure cost, median \pm SD[range], (euro) & $728 \pm 185[358-728]$ & $728 \pm 185[358-728]$ & 1 \\
\hline
\end{tabular}

ESD, conventional endoscopic submucosal dissection; ESTD, endoscopic submucosal tunnel dissection

Table 3 Anatomic and pathologic analyses of the resected specimens.

\begin{tabular}{|c|c|c|c|}
\hline & $\begin{array}{l}\text { ESD } \\
n=9\end{array}$ & $\begin{array}{l}\text { ESTD } \\
n=9\end{array}$ & $P$ \\
\hline En bloc resection, $\mathbf{n}(\%)$ & $8(88.9)$ & $9(100)$ & .49 \\
\hline Submucosal thickness, median \pm SD [range], $\mu \mathrm{m}$ & $884.7 \pm 224.3[330.5-1085.5]$ & $1307.1 \pm 421.3[728.5-1913.5]$ & .039 \\
\hline gastric site & $722.6 \pm 242.2[330.5-1009]$ & $728.0 \pm 502.1[727-1913.5]$ & \\
\hline esophageal site & $929.0 \pm 89.2[884.7-1086]$ & $1417.2 \pm 166.3[1307.1-1689]$ & \\
\hline
\end{tabular}

ESD, conventional endoscopic submucosal dissection; ESTD, endoscopic submucosal tunnel dissection 
Esophageal ESTD also gave rise to adverse events: two perforations of the muscularis, one during a vertical incision, and the other during a distal incision (both with the DualKnife ${ }^{\mathrm{TM}}$ ); both occurred during different procedures. In all of these cases, the perforations were no bigger than a few millimeters, unveiling only the longitudinal fibers of the muscularis, and could be treated by hemoclip. There were no gastric perforations.

During both ESD and ESTD, several arteriolar bleeding episodes occurred, and each one was successfully treated with the Dual$\mathrm{Knife}^{\mathrm{TM}}$ in soft coagulation mode. There was no significant bleeding that could not be endoscopically controlled or that necessitated the use of hemostatic clips.

\section{Use of minor instruments and cost of procedures}

In most cases, two knives were used for each procedure, be it for ESD $(66.6 \%)$ or ESTD $(77.8 \%)(P=.57) \bullet$ Table 2 . There was no significant difference in terms of knife changes. With knife changes taken into account, the median procedure cost was 728 Euros for ESD and 728 Euros for $\operatorname{ESTD}(P=1)$.

\section{Histologic analysis of surgical specimens}

All resection specimens were kept for analysis $\bullet$ Table 3, $\bullet$ Fig. 3 a, - Fig. 3 b. The overall rate of en bloc resection with healthy lateral margins (marker points visible) was $94.4 \%: 100 \%$ for ESTD and 88.9\% for ESD $(P=.49)$. On microscopic examination, the lateral zones appeared healthy despite coagulation artifacts at the edges. The deep resection margins were confined to the submucosal layer. Histologic specimens were thicker with resection by ESTD $(1307.1 \pm 421.3 \mu \mathrm{m}$ for ESTD $v s .884 .7 \pm 224.3 \mu \mathrm{m}$ for ESD) $(P=.039)$. In esophageal site, the depth of histologic specimens was of $929.0 \pm 89.2 \mu \mathrm{m}$ for ESD and of $1417.2 \pm 166.3 \mu \mathrm{m}$ for ESTD. In gastric site, the depth of histologic specimen was of $722.6 \pm$ $242.2 \mu \mathrm{m}$ for ESD and $728.0 \pm 502.1 \mu \mathrm{m}$ for ESTD.

\section{Discussion}

Over the last decade, new endoscopic resection techniques have made it possible to treat superficial tumors of the digestive tract in a minimally invasive fashion. Of these new techniques, ESD has shown its superiority over endoscopic mucosal resection in terms of en bloc resection, curative resection regardless of lesion size, and local recurrence [14-18]. Thus, ESD forms an obvious part of the oncologic treatment of patients, within well-documented indications [19-24].

This experimental study failed to find any difference between ESTD and ESD. Nevertheless, this may be due to the unexpected high rate of success with both techniques (ESTD and ESD) observed in the current study. That can be explained by the standardized procedure and reduced size of lesions, which were well demarcated and located in optimal anatomic conditions. Difficulties with positioning and stabilizing the endoscope hamper the continuation of submucosal dissection under visual control and augment the risk of bleeding and perforation. ESTD usefully avoids these problems of submucosal visibility: The endoscope is stabilized between the mucosa and muscular layers in the tunnel, permitting safe parallel access to the submucosal layer.

Regardless of which technique was used, the perforation rate was identical (22\%). All the perforations were small and millimeter, and occurred during esophageal procedures. It should be specified that perforations during ESTD occurred during the distal incision in one case and the proximal incision in the other. These

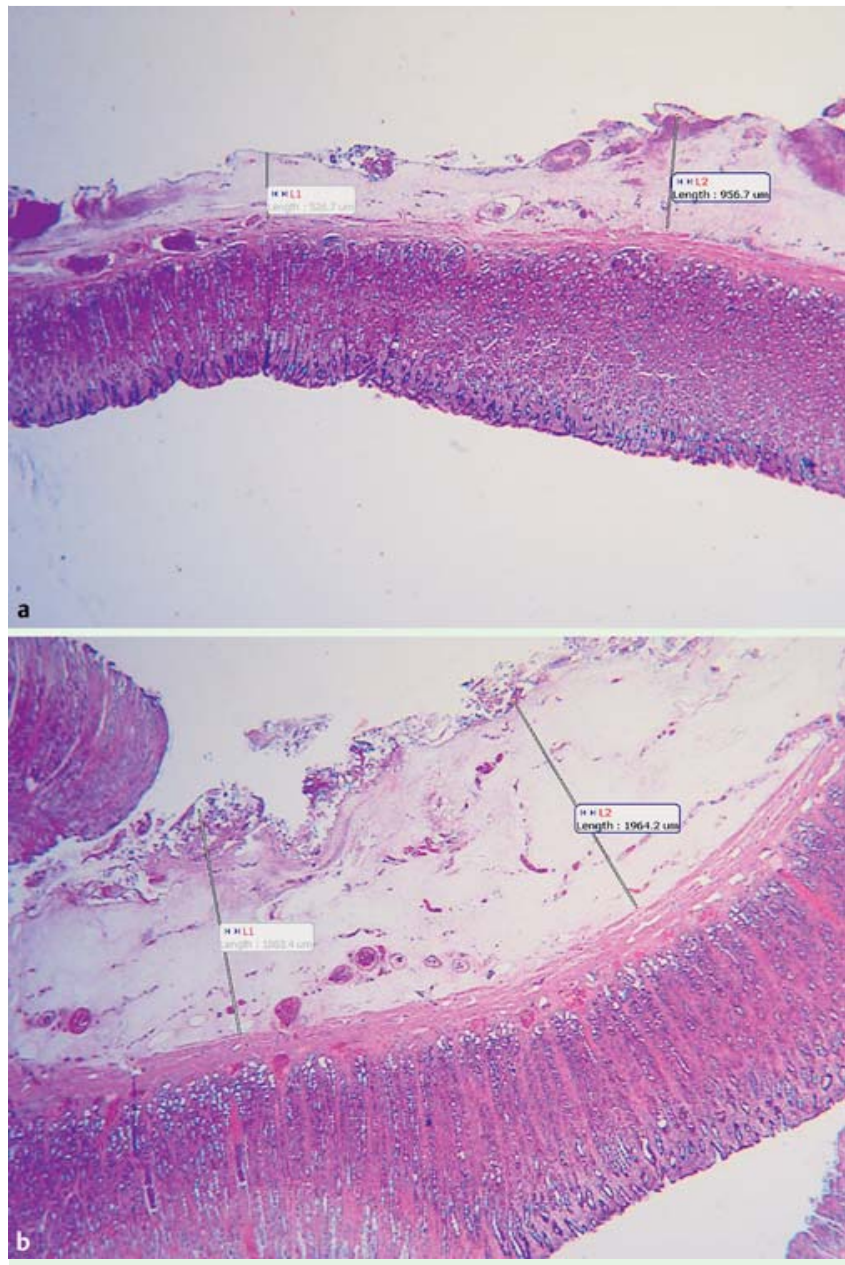

Fig.3 a Measure of the submucosal thickness of a conventional ESD specimen. $\mathbf{b}$ Measure of the submucosal thickness of an ESTD specimen with a significantly deeper submucosal space analyzed.

adverse events could, therefore, be attributed to a lack of experience with the technique and to insufficient initial lifting of the lesion. No perforation occurred during the dissection phase in the tunnel. This was not so with conventional ESD, which was complicated by two perforations during the same procedure, while the dissection was being performed. This suggests that the tunnel may improve safety during dissection, owing to better visibility [8-25]. Our perforation rate was higher than that observed in the literature for ESD $(<10 \%)[26,27]$. The perforations were, however, clinically inconsequential, and easily treated with clips. Several explanations are possible: An esophageal location may present greater risk due to a narrower luminal diameter and to a single working direction (anterograde), but also because of thinner submucosal layers in porcine experimental models $[4,28,29]$. The fact that the operators were less experienced than were the Japanese authors may also explain this difference $[3,30]$.

Although in this study there was no difference between the two techniques in terms of adverse events, improved visibility allowed the operators to better dissect fibers in the tunnel. First, vessels were more visible, and so, it was possible to coagulate them before continuing dissection. Second, indigo carmine staining of the submucosa in the tunnel rendered the border between the submucosa and muscularis distinctly visible, permitting safe submucosal dissection. Significant fibrosis can occur in the submucosal layer and cause difficulties in dissection, thus increasing 
the risk of perforations. These risks are increased by the flap of the lesion on the scope and the resection site without any dedicated material or techniques of traction (clip-with-line method, double-channel-scope method, outer route method) or by difficult anatomic position, which hampers visibility of fibers for dissection [31-33]. This phenomenon may also inhibit identification of the bleeding vessel, thus hindering accurate hemostasis. Therefore, ESTD would reduce these difficulties, given better stability of scope in the tunnel and a tangential resection plane relative to muscularis layer and therefore greater control of the knife during dissection. However, although in this study we were not confronted with massive bleeding in the tunnel, its management could be more challenging given the reduced working space.

Median procedure times, although not statistically significant, were longer for ESTD than for ESD. Yet it should be noted that the esophageal lesions resected by ESTD were larger than those resected by $\operatorname{ESD}(P=.028)$, which led to longer procedure times $(P=.2)$. Despite this, dissection speeds were quicker with ESTD, although the difference the techniques was significant only in the esophagus $(P=.03)$.This difference in procedure time may also be due to the fact that the two operators already possessed experience with ESD, both in porcine models and in humans, but not with the new ESTD technique.

In most cases, two knives are used for ESD or ESTD. Dual knife ${ }^{\mathrm{TM}}$ was preferably for marking, incision and dissection of the lesion, as well as coagulating vessels. With that knife, it was difficult to pull the lesion and incise the contours until reaching the tunnel. The IT2 knife ${ }^{\mathrm{TM}}$, in contrast, made dissection of the lesion contours comfortable because the ceramic ball at its end, which did not conduct current, facilitated traction of the lesion.

From a histologic perspective, there was no significant difference between the two techniques in terms of en bloc and R0 resection, although there appeared to be a trend in favor of ESTD. The rate of en bloc and R0 resection was similar to that observed in the literature (>90\%) $[14,15,17,18]$. Our study also revealed that ESTD enabled deeper dissection, submucosal thickness being statistically greater in $\operatorname{ESTD}(P=.039)$. In the gastric site, the thickness of the submucosal layer resectable was equivalent for ESTD and ESD. In the esophagus, the range of depth the histologic specimens was wider. To date, no data are available concerning the quality and thickness of the submucosal specimen obtained in ESD. To our knowledge, this study is the first to reveal histopathologically a significant gain in submucosal thickness with ESTD. That could be taken as a resection quality criterion that favors ESTD.

Our study presents several limitations. The number of procedures conducted with each technique was low with a potentially reduced statistical power of the work due to the unexpected high performance of ESD for en bloc resection. The results may have been influenced by the operators' experience with conventional ESD and their lack of experience with ESTD. Assessment of the lateral margins was handicapped by the absence of a real tumor, with lateral artifacts matching the marked areas impeding analysis. To assess the clinical consequences of adverse events, it would have been useful to monitor these animals over several days. Delayed hemorrhages occurring in the hours following the procedure could not, therefore, be assessed. Use of ESTD also has not been evaluated in some locations (e.g., angulus, subcardiac stomach). Indeed, in these locations, creating a tunnel would probably more difficult.

Two recent studies have demonstrated the feasibility of the technique for large lesions in the esophagus (mean $13.25 \mathrm{~cm}^{2}$ and average length $5.7 \mathrm{~cm}$ ) [10-11]. A case report has also been published about two cases in which an ulcerated and/or significantly fibrotic lesion in stomach was resected, eliminating the need for surgery in those patients [12]. Thus, ESTD could be an appropriate technique for resection of superficial lesions in esophagus and in some gastric sites (fundic and antral major curvature), regardless of lesion size. It also could be a rescue technique for ulcerated lesions, in specific cases.

In conclusion, ESTD appears to be feasible but not superior for resection of superficial esophageal/gastric lesions in porcine models, with promising technical and histologic results. The method does not seem to lead to any additional adverse events compared with conventional ESD. It may make it possible to significantly improve deep resection margins and to reduce procedure time in esophageal sites.

\section{Competing interests: None}

\section{Institutions}

'Endoscopie digestive, Hôpital L’Archet 2, Centre Hospitalier Universitaire, Nice, F-06202 Cedex 3, France

2 CERC, LBA UMRT24, Université Aix-Marseille, Faculté de médecine, Marseille, F-13916 Cedex20, France

${ }^{3}$ Gastro entérologie, Hôpital Nord, Assistance Publique des hôpitaux de Marseille, Marseille, F-13915 Cedex20, France

${ }^{4}$ Département d'anatomo-pathologie, Hôpital Pasteur, Centre Hospitalier Universitaire, Nice, F-06000, France

Chirurgie Digestive, Hôpital Nord, Assistance Publique des hôpitaux de Marseille, Marseille, F-13915 Cedex20, France

\section{Acknowledgements}

This study was funded by the "Fonds d'Aide à la Recherche et à l'Evaluation," a research and assessment support grant provided by the French national gastroenterology society in 2013 .

\section{References}

1 Ohkuwa M, Hosokawa K, Boku $N$ et al. New endoscopic treatment for intramucosal gastric tumors using an insulated-tip diathermic knife. Endoscopy 2001; 33: $221-226$

2 Kakushima N, Fujishiro M, Kodashima S et al. A learning curve for endoscopic submucosal dissection of gastric epithelial neoplasms. Endoscopy 2006; 38: $991-995$

3 Deprez PH, Bergman JJ, Meisner S et al. Current practice with endoscopic submucosal dissection in Europe: position statement from a panel of experts. Endoscopy 2010; 42: $853-858$

4 Berr F, Ponchon T, Neureiter D et al. Experimental endoscopic submucosal dissection training in a porcine model: learning experience of skilled Western endoscopists. Dig Endosc 2011; 23: 281 - 289

5 Teoh AY, Chiu PW, Wong SK et al. Difficulties and outcomes in starting endoscopic submucosal dissection. Surg Endosc 2010; 24: 1049-1054

6 Inoue $H$, Minami $H$, Kobayashi $Y$ et al. Peroral endoscopic myotomy (POEM) for esophageal achalasia. Endoscopy 2010; 42: 265 - 271

$7 \mathrm{Xu} M D$, Cai MY, Zhou PH et al. Submucosal tunneling endoscopic resection: a new technique for treating upper GI submucosal tumors originating from the muscularis propria layer (with videos). Gastrointest Endosc 2012; 75: 195-199

8 Gong W, Xiong Y, Zhi F et al. Preliminary experience of endoscopic submucosal tunnel dissection for upper gastrointestinal submucosal tumors. Endoscopy 2012; 44: 231 - 235

9 Yamamoto $H$. Endoscopic submucosal dissection for colorectal tumors. Front Gastrointes Res 2010; 27: 287-295

10 Pioche M, Mais L, Guillaud $O$ et al. Endoscopic submucosal tunnel dissection for large esophageal neoplastic lesions. Endoscopy 2013; 45: $1032-1034$

11 Linghu E, Feng $X$, Wang $X$ et al. Endoscopic submucosal tunnel dissection for large esophageal neoplastic lesions. Endoscopy 2013; 45: 6062 
12 Choi HS, Chun HJ, Seo MH et al. Endoscopic submucosal tunnel dissection salvage technique for ulcerative early gastric cancer. World J Gastroenterol 2014; 20: 9210-9214

13 Yamamoto H, Sekine Y, Higashizawa T et al. Successful en bloc resection of a large superficial gastric cancer by using sodium hyaluronate and electrocautery incision forceps. Gastrointest Endosc 2001; 54: 629632

14 Cao Y, Liao C, Tan A et al. Meta-analysis of endoscopic submucosal dissection versus endoscopic mucosal resection for tumors of the gastrointestinal tract. Endoscopy 2009; 41: $751-757$

15 Oda I, Saito D, Tada $M$ et al. A multicenter retrospective study of endoscopic resection for early gastric cancer. Gastric Cancer 2006; 9: 262 270

16 Sugimoto T, Okamoto M, Mitsuno $Y$ et al. Endoscopic submucosal dissection is an effective and safe therapy for early gastric neoplasms: a multicenter feasible study. J Clin Gastroenterol 2012; 46: 124-129

17 Saito Y, Uraoka T, Yamaguchi Yet al. A prospective, multicenter study of 1111 colorectal endoscopic submucosal dissections (with video). Gastrointest Endosc 2010; 72: 1217-1225

18 Akasaka T, Nishida T, Tsutsui $S$ et al. Short-term outcomes of endoscopic submucosal dissection (ESD) for early gastric neoplasm: multicenter survey by osaka university ESD study group. Dig Endosc 2011; 23: $73-77$

19 Hermanek P. The second English edition of the Japanese Classification of Gastric Carcinoma. A Western commentary. Gastric Cancer 1999; 2: $79-82$

20 Nonaka S, Oda I, Nakaya T et al. Clinical impact of a strategy involving endoscopic submucosal dissection for early gastric cancer: determining the optimal pathway. Gastric Cancer 2011; 14: 56-62

21 Fujishiro M, Kodashima S, Goto $O$ et al. Endoscopic submucosal dissection for esophageal squamous cell neoplasms. Dig Endosc 2009; 21: $109-115$

22 Seewald S, Ang TL, Gotoda T et al. Total endoscopic resection of Barrett esophagus. Endoscopy 2008; 40: 1016-1020
23 Yoshinaga S, Gotoda T, Kusano C et al. Clinical impact of endoscopic submucosal dissection for superficial adenocarcinoma located at the esophagogastric junction. Gastrointest Endosc 2008; 67: 202 - 209

24 Shimizu M, Nagata K, Yamaguchi H et al. Squamous intraepithelial neoplasia of the esophagus: past, present, and future. J Gastroenterol 2009; 44: $103-112$

25 Yoshizumi F, Yasuda K, Kawaguchi K et al. Submucosal tunneling using endoscopic submucosal dissection for peritoneal access and closure in natural orifice transluminal endoscopic surgery: a porcine survival study. Endoscopy 2009; 41: 707-711

26 Isomoto H, Yamaguchi N, Minami H et al. Management of complications associated with endoscopic submucosal dissection/endoscopic mucosal resection for esophageal cancer. Dig Endosc 2013; 25: 29-38

27 Hulagu S, Senturk O, Aygun C et al. Endoscopic submucosal dissection for premalignant lesions and noninvasive early gastrointestinal cancers. World J Gastroenterol 2011; 17: 1701 - 1709

28 Fujishiro M, Yahagi N, Kakushima $N$ et al. Outcomes of endoscopic submucosal dissection for colorectal epithelial neoplasms in 200 consecutive cases. Clin Gastroenterol Hepatol 2007; 5: 678 - 683 quiz 45

29 Oyama T, Tomori A, Hotta K et al. Endoscopic submucosal dissection of early esophageal cancer. Clin Gastroenterol Hepatol 2005; 3: 67-70

30 Repici A, Hassan C, De Paula Pessoa D et al. Efficacy and safety of endoscopic submucosal dissection for colorectal neoplasia: a systematic review. Endoscopy 2012; 44: 137-150

31 Lee BI. Debates on colorectal endoscopic submucosal dissection - traction for effective dissection: gravity is enough. Clin Endosc 2013; 46: $467-471$

32 Yonezawa J, Kaise M, Sumiyama K et al. A novel double-channel therapeutic endoscope ("R-scope") facilitates endoscopic submucosal dissection of superficial gastric neoplasms. Endoscopy 2006; 38: 1011 1015

33 Motohashi O, Nishimura K, Nakayama $N$ et al. Endoscopic submucosal dissection (two-point fixed ESD) for early esophageal cancer. Dig Endosc 2009; 21: 176-179 